\title{
Measuring gray matter and white matter damage in MS: why this is not enough
}

\author{
Christian Enzinger ${ }^{1,2 *}$ and Franz Fazekas ${ }^{1}$ \\ 1 Department of Neurology, Medical University of Graz, Graz, Austria \\ ${ }^{*}$ Correspondence: chris.enzinger@medunigraz.at \\ Edited by: \\ Maria Assunta Rocca, Vita-Salute San Raffaele University, Italy \\ Reviewed by: \\ Carsten Lukas, Ruhr-University Bochum, Germany \\ Antonio Giorgio, University of Siena, Italy
}

2 Division of Neuroradiology, Department of Radiology, Medical University of Graz, Graz, Austria

Keywords: multiple sclerosis, plasticity, adaptation, brain, function, gray matter, white matter, MRI

\section{INTRODUCTION}

Over the past years, progress in cerebral magnetic resonance imaging (MRI) technology has increased the possibilities to quantify MS-related tissue changes, starting from lesion assessment in the white matter (WM) to the quantification of microstructural changes of the whole brain (1). This was expected to give full insight into the causes of MS patients' deficits but despite all achievements, the current plethora of MRI metrics still provides no complete explanation for the clinical condition on a group (2) and even less so on an individual level. However, within the scope of personalized medicine, this remains an important goal to better understand and ultimately minimize the functional impact of MS-related tissue damage.

In this context, functional cerebral changes including adaptation and plasticity are strong contributors to the apparent clinical consequences of MS and are likely to explain part of the "morphologicalclinical gap" (3), notwithstanding ongoing controversies what patient deficits to consider and how to assess them. Against this background, we critically review the development and current state of techniques to assess gross MS-related morphologic damage and their contribution to understand the clinical consequences of MS (disability, and also cognitive problems and fatigue) and the obvious modulating roles of cerebral adaptation and plasticity as unraveled by functional MRI (fMRI). From existing data, we suggest that it is unlikely to ever achieve a satisfactory level of explanation and prediction of an individual patient's condition-based solely on morphologic information, although such insights might be better suited to define disease progression than clinical assessment.

\section{WHITE MATTER DAMAGE IN MS}

MS has traditionally been viewed as multifocal WM disease, and depicting lesions disseminated throughout the CNS using conventional MRI has become indispensable in early diagnosis and management (4). However, T2-weighted MRI lacks pathological specificity (5).

\section{FOCAL WHITE MATTER PATHOLOGY}

While the basic features of MS pathology constitute inflammation, demyelination in WM and GM, and diffuse neurodegeneration within the entire CNS, the individual components of the pathological spectrum vary quantitatively between early relapsing and late progressive MS (6). Moreover, remyelination of existing lesions may be extensive in a subset of patients and fail in others (6). All these components cannot be sufficiently assessed on T2-weighted MRI.

Compared to T2-hyperintense lesions, so-called "black holes" (severely and persistent hypointense lesions on T1-weighted MRI) have been shown to offer a more specific marker of matrix destruction and axonal loss (7). However, their definition is variable and strongly dependent on scanning parameters, which prohibits closer quantitation.

\section{NON-CONVENTIONAL MRI TO QUANTIFY LESIONAL WHITE MATTER DAMAGE}

A more refined insight into the composition of lesions may be gained by non-conventional techniques like magnetization transfer (MT) imaging (MTI), diffusion-weighted imaging (DWI), and diffusion tensor imaging (DTI).

MT-ratio (MTR) changes may precede the formation of active MS lesions by months, indicating changes in the macromolecular composition of pre-lesional WM long before a lesion becomes visible on conventional MRI (8). Once a lesion has formed, i.e., becomes apparent on T2weighted MRI, MTI may serve to follow the evolution and fate of affected parenchyma. Comparison with histopathology has shown good correlations with both demyelination and remyelination, and also just with fiber or neuronal density $(5,9)$. In a longitudinal trial, MTR-changes followed different temporal evolutions and were ongoing in different lesion regions for at least 3 years after lesion formation (10).

Diffusion-weighted imaging and DTI yield different insights. Diffusion measures the microscopic Brownian motion of water molecules, which is hindered by cellular structures (e.g., cell membranes and axonal cytoskeletons). In general, low-fractional anisotropy (FA) and high-mean diffusivity (MD) are found in MS lesions, but values are highly heterogeneous. Unfortunately, few studies investigated pathological correlates of DWI in MS. Surprisingly, at post-mortem, MD and FA correlated more strongly with myelin content than with axonal count and gliosis in one study (11).

\section{NON-CONVENTIONAL MRI TO QUANTIFY DIFFUSE WHITE MATTER DAMAGE}

Diffusion-weighted imaging/DTI and MTI played important roles in shaping the notion that MS not only consists of focal 
T2-lesions but also affects WM in a more widespread manner. Thus, normal appearing white matter (NAWM) in MS, in fact, is not normal, but demonstrates subtle microstructural abnormalities outside lesions. Histogram analyses have frequently been used to explore respective MRI metrics across the whole brain (12-14). Using MTI, MS patients consistently showed both lower average MTR and lower peak height (15) and respective DTI analyses demonstrated higher average MD, lower histogram peak height $\mathrm{MD}$, and lower average FA $(14,16)$ compared to healthy controls, respectively. Lower FA values close to and higher values far from MS lesions suggest Wallerian degeneration (17), but MD and FA of NAWM only partially correlate with the extent and severity of focal lesions, indicating other factors like astrocytic hyperplasia, patchy edema, perivascular infiltration, demyelination, and axonal loss also contribute to such abnormalities (14).

\section{STRATEGIC LOCATION OF WHITE MATTER DAMAGE}

Assessing the strategic location of WM damage using lesion probability maps (LPM) or diffusion-based tractography (DBT) represents another promising approach to improve upon morphologicalclinical correlations. Thus, LPM in different MS phenotypes revealed associations between specific neurologic and cognitive deficits with lesion accumulation in distinct, anatomically plausible, regions, but only to limited extent (18). Using tract-based spatial statistics [TBSS; a fully automated, wholebrain diffusion analysis method (14)] to identify loci where reduced WM-tract FA predicted impaired cognitive performance in MS patients, cognitively relevant tract localizations only partially overlapped with areas of high-lesion probability, but identified tract localizations were found to interconnect cortical regions involved in cognitive processing (19). Thus, there is evidence that abnormalities in strategic brain WM tracts contribute to cognitive impairment in MS, but the identified regions vary between studies and it is unlikely that such analyses could be performed on an individual level.

\section{GRAY MATTER DAMAGE IN MS GRAY MATTER PATHOLOGY}

Autopsy studies have demonstrated that MS is also associated with focal lesions in and diffuse demyelination of GM $(20,21)$. The depiction of these kinds of MS-related damage has been and still is a challenge for MRI.

\section{FOCAL GRAY MATTER DAMAGE}

Intrinsically, low-myelin densities in the cortex (where demyelination generates little contrast), the often small size of lesions, and partial volume effects of cerebrospinal fluid impede the detection of cortical GM lesions on conventional MRI (21). Introduction of the double-inversion recovery (DIR) sequence with superior sensitivity compared to $\mathrm{T} 2$ - and fluidattenuated-inversion-recovery (FLAIR) sequences improved this situation, but post-mortem comparison showed that $80 \%$ of lesions still remain undetected $(20,21)$. Interestingly, MRI-visible cortical lesions do not differ from MRI-invisible lesions in their pathological profiles (20). Combination with other sequences such as phase-sensitive inversion recovery and T1-weighted 3D-spoiled gradient-recalled echo and ultra-high-field scanners might help to partly overcome these problems $(20,22)$, but this approach is not realizable in clinical settings. Importantly, despite representing the commonest lesion type, subpial cortical lesions largely escape detection by MRI (20). Deep GM pathology is somewhat easier to depict than cortical GM pathology as lesions in deep GM structures, spinal cord, and hippocampus are generally mixed GM/WM lesions and slightly more inflammatory (21).

\section{DIFFUSE GRAY MATTER DAMAGE}

Cortical thickness is reduced in MS (23) and a mean cortical thinning of $10 \%$ has been found independently of cortical lesions, suggesting mechanisms besides cortical demyelination contribute to cortical atrophy (21). A recent study combining post-mortem imaging and histopathology to explore the underpinnings of cortical atrophy identified neuronal density, neuronal size, and axonal density as predictors of cortical GM volume in long-standing MS (24). GM constitutes about 65\% of brain parenchymal tissue, and atrophy of
GM largely drives whole-brain atrophy in MS (20). GM atrophy in MS occurs both at global and regional level and can be quantitated using MRI. It also does not directly correlate with the number of WM lesions and diffuse NAWM damage, suggesting partially independent pathological processes (22). Unfortunately, GM volume measures are inherently non-specific and reveal little about the exact cause of tissue injury (20).

\section{NON-CONVENTIONAL MRI TO OUANTIFY GRAY MATTER DAMAGE}

Analogous to but less frequent than respective WM studies, DWI/DTI and MTI have also been used to quantify GM damage in MS. Consistent with differences in pathology, DTI detected higher MD and FA in cortical lesions than in WM lesions of MS patients (22). New approaches to study cortical MTR changes include segmentation of the cortex to obtain separate information on outer and inner bands, where the lowest outer cortical MTR was seen in secondary progressive MS, consistent with post-mortem findings of more extensive subpial pathology in this group (25).

\section{STRATEGIC LOCATION OF GRAY MATTER DAMAGE}

Extensive GM involvement has been associated with cognitive decline, motor deficits, fatigue, painful syndromes, and ocular motility disturbances in MS. In this respect, the thalamus has been highlighted, as it relays sensory information to higher cortical centers that influence cognition (26). A strategic significance has also been demonstrated for the hippocampus, as lesions in this area strongly correlate with impaired visuospatial memory and processing speed (20, 21). Further, using LPMs of DIR images, cortical MS lesions have been demonstrated to be mainly distributed in the frontal and temporal lobes, with prominent involvement of motor and anterior cingulate cortices (27).

\section{CONTRIBUTION OF WHITE AND GRAY MATTER DAMAGE TO EXPLAINING CLINICAL DEFICITS}

All the above techniques have greatly enhanced our understanding of the complexity of MS tissue damage and provide possibilities to assess the structural changes 
associated with MS in ever more detail. At group level, several discussed metrics alone or in combination have shown good correlation with measures of disability or cognition [e.g., Ref. (28)] and even simple MRI markers appear to be an excellent surrogate for treatment response (29). However, these complex insights have not transformed into a sufficient capacity to explain or even predict a patient's functioning and clinical deficits, and are far from having reached utility in daily clinical practice. We suggest that the reasons for this are not only the complexity of MS-related damage - which is certainly even much greater than addressed above (e.g., consider the role of other CNS compartments such as the spinal cord) - but rather the individual variability in processes of plasticity and adaptation.

\section{FUNCTIONAL MRI AS ONE APPROACH TO GAIN FUNCTIONAL INFORMATION}

Functional MRI studies of visual, cognitive, and motor systems consistently demonstrated functional changes in all MS phenotypes, characterized by altered activation of regions normally devoted to performance of a task or recruitment of additional areas compared to healthy subjects $(3,22,30$, $31)$. Given the correlation between functional and structural abnormalities (30), the former appear to partly limit the functional consequences of structural damage in MS. fMRI abnormalities already occur in CIS, but differences in activation patterns between phenotypes are striking (32, 33), suggesting profound changes in the functional organization of the MS brain with disease progression. Final exhaustion of adaptive capacity may constitute one key factor for unfavorable clinical evolution or cognitive decline, although the decisive factors driving transition from adaption to maladaptation are unknown. However, maladaptation in MS is not only the consequence of the final exhaustion of adaptive plasticity but it may also be expressed in early stages of the disease as enhanced functional connectivity (34). While patients with "long-term low disability MS" may functionally withstand considerable amounts of brain tissue damage, others already suffer from severe disability (35). Individual differences in brain reserve and cognitive reserve thereby might play a role (36-38). Combining MRI measures of structural damage with those of abnormal functional and structural connectivity (3) using resting state fMRI $(13,39)$ appears promising to further elucidate such relationships at group level, but this would necessitate prospective longitudinal studies (40) with long-term follow-up, ideally in multi-center settings (41).

\section{CONCLUSION AND FUTURE DIRECTIONS}

Despite increasing level of detail, morphological insights using MRI by nature only allow assessment of (micro)structural disease-related processes in MS-brains. fMRI enabled detection of paralleling adaptive cerebral changes, which - besides the stage and dynamics of the disease most likely are also modulated by individual differences ("functional reserve"). Interpretations of such changes are corroborated and usefully augmented by concomitant assessment of morphological MRI changes. Yet, measurement of brain damage by structural MRI alone clearly does not suffice to comprehensively appreciate the consequences of the disease, although it is ideal for specific questions (e.g., assessment of disease activity, remyelination, or evolution of atrophy). To better understand mechanisms of functional adaption in MS, longitudinal studies including (micro)structural and functional MRI in large registries of early MS followed for many years are needed. Whether this will finally allow judging capacity for adaption at the individual level remains unclear.

\section{REFERENCES}

1. Filippi M, Rocca MA, De Stefano N, Enzinger C, Fisher E, Horsfield MA, et al. Magnetic resonance techniques in multiple sclerosis: the present and the future. Arch Neurol (2011) 68:1514-20. doi:10.1001/archneurol.2011.914

2. Goodin DS. Magnetic resonance imaging as a surrogate outcome measure of disability in multiple sclerosis: have we been overly harsh in our assessment? Ann Neurol (2006) 59:597-605. doi: 10.1002/ana.20832

3. Tomassini V, Matthews PM, Thompson AJ, Fuglo D, Geurts JJ, Johansen-Berg H, et al. Neuroplasticity and functional recovery in multiple sclerosis. Nat Rev Neurol (2012) 8:635-46. doi:10.1038/ nrneurol.2012.179

4. Montalban X, Tintore M, Swanton J, Barkhof F, Fazekas F, Filippi M, et al. MRI criteria for MS in patients with clinically isolated syndromes. Neurology (2010) 74:427-34. doi:10.1212/WNL. 0b013e3181cec45c
5. Filippi M, Rocca MA, Barkhof F, Bruck W, Chen JT, Comi G, et al. Association between pathological and MRI findings in multiple sclerosis. Lancet Neurol (2012) 11:349-60. doi:10.1016/ S1474-4422(12)70003-0

6. Kutzelnigg A, Lassmann H. Pathology of multiple sclerosis and related inflammatory demyelinating diseases. Handb Clin Neurol (2014) 122:15-58. doi:10.1016/B978-0-444-52001-2.00002-9

7. van Walderveen M, Kamphorst W, Scheltens $\mathrm{P}$, van Waesberghe J, Ravid R, Valk J, et al. Histopathologic correlate of hypointense lesions on T1-weighted spin-echo MRI in multiple sclerosis. Neurology (1998) 50:1282-8. doi:10.1212/ WNL.50.5.1282

8. Fazekas F, Ropele S, Enzinger C, Seifert T, StrasserFuchs S. Quantitative magnetization transfer imaging of pre-lesional white-matter changes in multiple sclerosis. Mult Scler (2002) 8:479-84. doi:10.1191/1352458502ms860oa

9. Chen JT, Kuhlmann T, Jansen GH, Collins DL, Atkins HL, Freedman MS, et al. Voxel-based analysis of the evolution of magnetization transfer ratio to quantify remyelination and demyelination with histopathological validation in a multiple sclerosis lesion. Neuroimage (2007) 36:1152-8. doi:10.1016/j.neuroimage.2007.03.073

10. Chen JT, Collins DL, Atkins HL, Freedman MS, Arnold DL, Canadian MS/BMT Study Group. Magnetization transfer ratio evolution with demyelination and remyelination in multiple sclerosis lesions. Ann Neurol (2008) 63:254-62. doi:10.1002/ana.21302

11. Schmierer K, Wheeler-Kingshott CA, Boulby PA, Scaravilli F, Altmann DR, Barker GJ, et al. Diffusion tensor imaging of post mortem multiple sclerosis brain. Neuroimage (2007) 35:467-77. doi:10.1016/j.neuroimage.2006.12.010

12. Parisi L, Rocca MA, Valsasina P, Panicari L, Mattioli F, Filippi M. Cognitive rehabilitation correlates with the functional connectivity of the anterior cingulate cortex in patients with multiple sclerosis. Brain Imaging Behav (2014) 8:387-93. doi:10. 1007/s11682-012-9160-9

13. Enzinger C, DeLuca J. Large-scale neuronal network dysfunction in multiple sclerosis?: evidence from resting-state fMRI. Neurology (2012) 79:1416-7. doi:10.1212/WNL.0b013e31826d600d

14. Sbardella E, Tona F, Petsas N, Pantano P. DTI measurements in multiple sclerosis: evaluation of brain damage and clinical implications. Mult Scler Int (2013) 2013:671730. doi:10.1155/2013/ 671730

15. Loitfelder M, Filippi M, Rocca M, Valsasina P, Ropele S, Jehna $\mathrm{M}$, et al. Abnormalities of resting state functional connectivity are related to sustained attention deficits in MS. PLoS One (2012) 7:e42862. doi:10.1371/journal.pone.0042862

16. Inglese M, Bester M. Diffusion imaging in multiple sclerosis: research and clinical implications. NMR Biomed (2010) 23:865-72. doi:10. 1002/nbm.1515

17. Rocca MA, Valsasina P, Meani A, Falini A, Comi G, Filippi M. Impaired functional integration in multiple sclerosis: a graph theory study. Brain Struct Funct (2014). doi:10.1007/s00429-014-0896-4

18. Kincses ZT, Ropele S, Jenkinson M, Khalil M, Petrovic K, Loitfelder M, et al. Lesion probability mapping to explain clinical deficits and cognitive 
performance in multiple sclerosis. Mult Scler (2011) 17:681-9. doi:10.1177/1352458510391342

19. Dineen RA, Vilisaar J, Hlinka J, Bradshaw CM, Morgan PS, Constantinescu CS, et al. Disconnection as a mechanism for cognitive dysfunction in multiple sclerosis. Brain (2009) 132:239-49. doi:10.1093/brain/awn275

20. Geurts JJ, Calabrese M, Fisher E, Rudick RA. Measurement and clinical effect of grey matter pathology in multiple sclerosis. Lancet Neurol (2012) 11:1082-92. doi:10.1016/S1474-4422(12)70230-2

21. Klaver R, De Vries HE, Schenk GJ, Geurts JJ. Grey matter damage in multiple sclerosis: a pathology perspective. Prion (2013) 7:66-75. doi:10. 4161/pri.23499

22. Filippi M. MRI measures of neurodegeneration in multiple sclerosis: implications for disability, disease monitoring, and treatment. J Neurol (2015) 262:1-6. doi:10.1007/s00415-014-7340-9

23. Steenwijk MD, Daams M, Pouwels PJ, Balk LJ, Tewarie PK, Killestein J, et al. What explains gray matter atrophy in long-standing multiple sclerosis? Radiology (2014) 272:832-42. doi:10.1148/radiol. 14132708

24. Popescu V, Klaver R, Voorn P, Galis-de Graaf Y, Knol D, Twisk J, et al. What drives MRI-measured cortical atrophy in multiple sclerosis? Mult Scler (2015). doi:10.1177/1352458514562440

25. Samson RS, Cardoso MJ, Muhlert N, Sethi V, Wheeler-Kingshott CA, Ron $\mathrm{M}$, et al. Investigation of outer cortical magnetisation transfer ratio abnormalities in multiple sclerosis clinical subgroups. Mult Scler (2014) 20:1322-30. doi:10. $1177 / 1352458514522537$

26. Minagar A, Barnett MH, Benedict RH, Pelletier D, Pirko I, Sahraian MA, et al. The thalamus and multiple sclerosis: modern views on pathologic, imaging, and clinical aspects. Neurology (2013) 80:210-9. doi:10.1212/WNL.0b013e31827b910b

27. Calabrese M, Battaglini M, Giorgio A, Atzori M, Bernardi V, Mattisi I, et al. Imaging distribution and frequency of cortical lesions in patients with multiple sclerosis. Neurology (2010) 75:1234-40. doi:10.1212/WNL.0b013e3181f5d4da

28. Mesaros S, Rocca MA, Kacar K, Kostic J, Copetti M, Stosic-Opincal T, et al. Diffusion tensor MRI tractography and cognitive impairment in multiple sclerosis. Neurology (2012) 78:969-75. doi:10. 1212/WNL.0b013e31824d5859

29. Sormani MP, Bruzzi P. MRI lesions as a surrogate for relapses in multiple sclerosis: a metaanalysis of randomised trials. Lancet Neurol (2013) 12:669-76. doi:10.1016/S1474-4422(13)70103-0
30. Filippi M, Rocca MA. Functional MR imaging in multiple sclerosis. Neuroimaging Clin N Am (2009) 19:59-70. doi:10.1016/j.nic.2008.08.004

31. Filippi M, Grossman RI. MRI techniques to monitor MS evolution: the present and the future. Neurology (2002) 58:1147-53. doi:10.1212/WNL. 58.8 .1147

32. Loitfelder M, Fazekas F, Petrovic K, Fuchs S, Ropele S, Wallner-Blazek M, et al. Reorganization in cognitive networks with progression of multiple sclerosis: insights from fMRI. Neurology (2011) 76:526-33. doi:10.1212/WNL.0b013e31820b75cf

33. Rocca MA, Colombo B, Falini A, Ghezzi A, Martinelli V, Scotti G, et al. Cortical adaptation in patients with MS: a cross-sectional functional MRI study of disease phenotypes. Lancet Neurol (2005) 4:618. doi:10.1016/S1474-4422(05)70171-X

34. Hawellek DJ, Hipp JF, Lewis CM, Corbetta M, Engel AK. Increased functional connectivity indicates the severity of cognitive impairment in multiple sclerosis. Proc Natl Acad Sci U S A (2011) 108:19066-71. doi:10.1073/pnas.1110024108

35. Strasser-Fuchs S, Enzinger C, Ropele S, Wallner M, Fazekas F. Clinically benign multiple sclerosis despite large T2 lesion load: can we explain this paradox? Mult Scler (2008) 14:205-11. doi:10. $1177 / 1352458507082354$

36. Pinter D, Sumowski J, DeLuca J, Fazekas F, Pichler A, Khalil M, et al. Higher education moderates the effect of T2 lesion load and third ventricle width on cognition in multiple sclerosis. PLoS One (2014) 9:e87567. doi:10.1371/journal.pone.0087567

37. Sumowski JF, Rocca MA, Leavitt VM, Dackovic J, Mesaros S, Drulovic J, et al. Brain reserve and cognitive reserve protect against cognitive decline over 4.5 years in MS. Neurology (2014) 82:1776-83 doi:10.1212/WNL.0000000000000433

38. Amato MP, Razzolini L, Goretti B, Stromillo ML Rossi F, Giorgio A, et al. Cognitive reserve and cortical atrophy in multiple sclerosis: a longitudinal study. Neurology (2013) 80:1728-33. doi:10.1212/ WNL.0b013e3182918c6f

39. Rocca MA, Valsasina P, Martinelli V, Misci P, Falini A, Comi G, et al. Large-scale neuronal network dysfunction in relapsing-remitting multiple sclerosis. Neurology (2012) 79:1449-57. doi:10.1212/WNL. 0b013e31826d5f10

40. Loitfelder M, Fazekas F, Koschutnig K, Fuchs S, Petrovic K, Ropele S, et al. Brain activity changes in cognitive networks in relapsing-remitting multiple sclerosis - insights from a longitudinal fMRI study. PLoS One (2014) 9:e93715. doi:10.1371/journal. pone.0093715
41. Rocca MA, Valsasina P, Hulst HE, Abdel-Aziz K, Enzinger C, Gallo A, et al. Functional correlates of cognitive dysfunction in multiple sclerosis: a multicenter fMRI Study. Hum Brain Mapp (2014) 35:5799-814. doi:10.1002/hbm.22586

Conflict of Interest Statement: This opinion article was drafted in the absence of any commercial or financial relationships that could be construed as a potential conflict of interest. Declaration of financial relationships and other activities: Dr. Christian Enzinger has received funding for travel and speaker honoraria from Biogen Idec, Bayer Schering Pharma, Merck Serono, Novartis Genzyme, and Teva Pharmaceutical Industries Ltd./sanofi-aventis; research support from Merck Serono, Biogen Idec., and Teva Pharmaceutical Industries Ltd./sanofi-aventis; serving on scientific advisory boards for Bayer Schering Pharma, Biogen Idec, Genzyme, Merck Serono, Novartis, and Teva Pharmaceutical Industries Ltd./sanofi-aventis; academic editor for PLOSOne. Dr. Franz Fazekas serves on scientific advisory boards for Bayer-Schering, Biogen Idec, Genzyme, Merck Serono, Pfizer, Novartis, and Teva Pharmaceutical Industries Ltd.; serves on the editorial boards of Cerebrovascular Diseases, Multiple Sclerosis, the Polish Journal of Neurology and Neurosurgery, Stroke, and the Swiss Archives of Neurology and Psychiatry; and has received speaker honoraria and support from Biogen Idec, Bayer Schering, Merck Serono, Novartis, Pfizer, Sanofi-Aventis, Shire and Teva Pharmaceutical Industries Ltd.

Received: 30 January 2015; paper pending published: 11 February 2015; accepted: 27 February 2015; published online: 17 March 2015

Citation: Enzinger $C$ and Fazekas F (2015) Measuring gray matter and white matter damage in MS: why this is not enough. Front. Neurol. 6:56. doi: 10.3389/fneur.2015.00056

This article was submitted to Multiple Sclerosis and Neuroimmunology, a section of the journal Frontiers in Neurology.

Copyright (C) 2015 Enzinger and Fazekas. This is an openaccess article distributed under the terms of the Creative Commons Attribution License (CC BY). The use, distribution or reproduction in other forums is permitted, provided the original author(s) or licensor are credited and that the original publication in this journal is cited, in accordance with accepted academic practice. No use, distribution or reproduction is permitted which does not comply with these terms. 